\title{
Acoustic Analysis: An Objective Tool in Evaluating Patients with Voice Disorders
}

\author{
Susan P Chacko ${ }^{1}$, Anagha A Joshi ${ }^{2}$, Varun Dave ${ }^{3}$
}

\begin{abstract}
Introduction: Acoustic measurements are the most widely studied of all voice measures. Our study aimed to determine the accuracy of acoustic analysis as an objective tool for evaluating patients with voice disorders.

Materials and methods: A descriptive cross-sectional study with a total of 73 patients was analyzed using Dr Speech software.

Results: When the vocal parameters were compared between normal subjects and patients with vocal cord diseases, significant differences were seen in most of the parameters like jitter, shimmer, Normalized Noise Energy (NNE), Harmonic to Noise Ratio (HNR), Signal to Noise Ratio (SNR) along with a statistically significant difference in voice quality scores like harsh voice, hoarse voice, and breathy voice.

Conclusion: Hence, from our study, it can be said that acoustic analysis is a reliable objective tool for evaluating voice disorders.

Keywords: Dysphonia, Hoarseness, Multiparametric acoustic analysis, Objective voice assessment, Quality of voice, Vocal cord dysfunction. International Journal of Phonosurgery \& Laryngology (2020): 10.5005/jp-journals-10023-1189
\end{abstract}

\section{INTRODUCTION}

A comprehensive evaluation of voice is the first step in the appropriate management of voice disorders. Various techniques have been used to assess the patient's voice quality. One of them is the auditory perceptual analysis; however, this is a subjective assessment that gives different results depending on the experience of the practitioner involved. Therefore, an objective assessment of voice is required which is capable of measuring several acoustic parameters.

Acoustic measurements are the most widely studied of all voice measures. It aims to quantify and characterize a sound signal in a noninvasive manner and has the advantage of describing the voice objectively. By combining the various parameters, it is possible to distinguish between normal and pathological voice or even suggest the pathology. These tools reduce the degree of subjectivity of perceptual analysis.

Commonly used parameters in applications of acoustic analysis are the fundamental frequency $(F 0)$, jitter, shimmer, Harmonic to Noise Ratio (HNR), Signal to Noise Ratio (SNR), Normalized Noise Energy (NNE), and frequency formants. In literature, measurements of FO disturbance, jitter, and shimmer have proven to be useful in describing the vocal characteristics.

\section{Materials and Methods}

This research was a descriptive cross-sectional study. A total of 73 patients were analyzed. This study included normal patients, as well as patients with laryngeal disorder and the age considered, was between 15 years and 65 years.

Patients with cognitive or neurologic alterations were excluded from the study.

The data were collected at the time of the assessment of the patient before the vocal treatment was initiated. A detailed history of personal and vocal history was taken. Personal information like age, gender, and profession was taken. Dr Speech software, Tiger DRS, Inc. was used with a microphone for objective voice analysis
${ }^{1-3}$ Department of ENT and Head and Neck Surgery, Lokmanya Tilak Municipal Medical College and General Hospital, Mumbai, Maharashtra, India

Corresponding Author: Susan P Chacko, Department of ENT and Head and Neck Surgery, Lokmanya Tilak Municipal Medical College and General Hospital, Mumbai, Maharashtra, India, Phone: +91 9594900147, e-mail: dr.susanchacko@gmail.com

How to cite this article: Chacko SP, Joshi AA, Dave V. Acoustic Analysis: An Objective Tool in Evaluating Patients with Voice Disorders. Int J Phonosurg Laryngol 2020;10(2):29-32.

Source of support: Nil

Conflict of interest: None

and the voices were recorded in a place where the ambient noise level was $<50 \mathrm{~dB}$.

The patients were asked to produce a sustained vowel/a/, in a frequency and intensity considered normal and comfortable by the patient. Each speaker sat comfortably with a microphone $10 \mathrm{~cm}$ away from the mouth. The sampling frequency used for recording these signals was $44100 \mathrm{~Hz}$ at a sampling rate with 16 bits per sample.

Subsequently, the voices were edited using Dr Speech software, windows 95-10, version 4.61U304. The two initial and final seconds of the emission of the vowel were eliminated due to the greater irregularity in these passages, while the minimum time of 4 seconds for each emission was preserved.

The patients were divided into the following four categories

- Healthy larynx.

- Vocal fold polyp.

- Unilateral vocal fold palsy.

- Vocal fold nodules.

\section{Results}

In our study, parameters were compared between normal and diseased groups of patients.

(c) The Author(s). 2020 Open Access This article is distributed under the terms of the Creative Commons Attribution 4.0 International License (https://creativecommons. org/licenses/by-nc/4.0/), which permits unrestricted use, distribution, and non-commercial reproduction in any medium, provided you give appropriate credit to the original author(s) and the source, provide a link to the Creative Commons license, and indicate if changes were made. The Creative Commons Public Domain Dedication waiver (http://creativecommons.org/publicdomain/zero/1.0/) applies to the data made available in this article, unless otherwise stated. 
- The comparison was done using various acoustic parameters between normal and diseased groups of patients, significant differences were seen in all parameters except habitual frequency $(\mathrm{Hz}), \mathrm{s} / \mathrm{z}$ ratio, and $\mathrm{F} 0$ tremor. The data were compared using mean $\pm S D$. The statistical significance was calculated using ANOVA one-way and Scheffe post hoc test (Table 1).

- The comparison was done between normal male and diseased male groups of patients, the vocal parameter habitual frequency showed significant differences between normal subjects and patients with vocal cord nodules. The data were compared using mean $\pm S D$. The statistical significance was calculated using the Scheffe post hoc test (Table 2).

- The comparison was done between normal female and diseased female groups of patients; the vocal parameter habitual frequency did not any show a statistical difference. The data were compared using mean \pm SD. The statistical test was done using ANOVA one-way and Scheffe post hoc test (Table 3).

- The comparison of voice quality was done between normal and diseased groups of patients; significant differences were seen in all parameters except gender. The data were compared using the number (\%). The statistical significance was calculated using Chi-square test (Table 4).

\section{Discussion}

In literature, many studies are applying acoustic analysis as an objective tool to substantiate correlation with voice disorders.
Ziwei et al. ${ }^{1}$ quoted that the selection of parameters in objective voice assessment should meet three fundamental principles:

- Must have pathophysiological significance.

- Variation in assessment results should be consistent with the severity of voice disorders.

- Normal and dysphonic voices can be effectively defined by values of the parameters.

According to Teixeira et al., ${ }^{2}$ normal subjects have lower jitter and shimmer, whereas HNR has no significant difference between normal and pathologic group, in our study statistical significance was seen with jitter and shimmer and HNR.

In literature, jitter and shimmer gave relatively higher values evidencing pathological condition. ${ }^{3}$ Our data also gave similar findings.

According to Teixeira and Fernandes study, ${ }^{4}$ the HNR parameter did not show strong discriminant measurements, whereas jitter and shimmer seemed to be relevant parameters to be used in the diagnosis of dysphonia pathologies. In our study, HNR did show statistical significance with a $p=0.014$ (Table 1) along with jitter and shimmer.

Teixeira and Fernandes compared the variability of voice in both genders. ${ }^{5}$ Only the jitter parameter showed statistically significant differences between male and female voices, being higher for male voices, and the remaining parameters there are no statistical pieces of evidence of differences for male and female voices. In our study,

Table 1: Comparison of acoustic parameters between normal and diseased groups of patients

\begin{tabular}{|c|c|c|c|c|c|c|}
\hline $\begin{array}{l}\text { Parameter } \\
\text { diagnosis }\end{array}$ & $\begin{array}{l}\text { Normal (I) } \\
(n=23)\end{array}$ & $\begin{array}{l}\text { Polyp (II) } \\
(n=21)\end{array}$ & $\begin{array}{l}\text { Palsy (III) } \\
(n=17)\end{array}$ & $\begin{array}{l}\text { Nodules (IV) } \\
(n=13)\end{array}$ & $\begin{array}{l}\text { F test value, significance, } \\
\text { and } p \text { value }\end{array}$ & $\begin{array}{l}\text { Scheffe post hoc test, } \\
\text { significant difference } \\
\text { between pairs of means }\end{array}$ \\
\hline Age & $27.26 \pm 3.47$ & $37.38 \pm 12.32$ & $36.00 \pm 12.93$ & $39.08 \pm 14.35$ & $F=4.7, \mathrm{~S}, p=0.005$ & $1-2,1-4$ \\
\hline $\begin{array}{l}\text { Habitual } \\
\text { frequency }(\mathrm{Hz})\end{array}$ & $181.06 \pm 48.92$ & $178.80 \pm 63.94$ & $182.56 \pm 56.21$ & $233.85 \pm 65.29$ & $F=3.0, \mathrm{~S}, p=0.036$ & NS \\
\hline Jitter (\%) & $0.21 \pm 0.08$ & $0.90 \pm 0.81$ & $1.49 \pm 2.41$ & $0.91 \pm 0.91$ & $F=3.3, \mathrm{~S}, p=0.026$ & $1-3$ \\
\hline Shimmer (\%) & $2.36 \pm 1.09$ & $5.50 \pm 2.67$ & $5.17 \pm 3.02$ & $3.95 \pm 2.54$ & $F=7.7, \mathrm{~S}, p<0.001$ & $1-2,1-3$ \\
\hline MPT (sec) & $17.21 \pm 3.53$ & $6.77 \pm 5.28$ & $5.08 \pm 4.18$ & $4.51 \pm 2.35$ & $F=43.1, S, p<0.001$ & $1-2,1-3,1-4$ \\
\hline NNE & $-13.74 \pm 4.61$ & $-3.78 \pm 3.18$ & $-3.73 \pm 3.03$ & $-4.40 \pm 3.24$ & $F=37.9, \mathrm{~S}, p<0.001$ & $1-2,1-3,1-4$ \\
\hline HNR & $20.84 \pm 4.68$ & $16.07 \pm 4.31$ & $17.40 \pm 5.85$ & $19.63 \pm 5.50$ & $F=3.8, S, p=0.014$ & $1-2$ \\
\hline SNR & $19.89 \pm 4.52$ & $14.98 \pm 4.06$ & $16.14 \pm 5.67$ & $18.39 \pm 5.26$ & $F=4.4, \mathrm{~S}, p=0.007$ & $1-2$ \\
\hline s/z ratio & $0.77 \pm 0.22$ & $3.64 \pm 7.71$ & $1.56 \pm 2.67$ & $1.67 \pm 1.64$ & $F=1.7, \mathrm{NS}, p=0.18$ & NS \\
\hline F0 tremor & $4.12 \pm 3.09$ & $5.30 \pm 4.83$ & $4.57 \pm 4.23$ & $5.22 \pm 4.91$ & $F=0.4, \mathrm{NS}, p=0.8$ & NS \\
\hline Amp tremor & $6.06 \pm 4.43$ & $5.11 \pm 5.02$ & $3.67 \pm 3.06$ & $2.00 \pm 1.67$ & $F=3.3, \mathrm{~S}, p=0.026$ & $1-4$ \\
\hline
\end{tabular}

Table 2: Comparison between normal male and diseased group of male patients

\begin{tabular}{|c|c|c|c|c|c|c|}
\hline Parameter & $\operatorname{Normal}(n=11)$ & Polyp $(n=12)$ & Palsy $(n=7)$ & Nodule $(n=4)$ & $p$ value & $\begin{array}{l}\text { Scheffe post hoc } \\
\text { test, significant } \\
\text { difference between } \\
\text { pairs of means }\end{array}$ \\
\hline $\begin{array}{l}\text { Habitual } \\
\text { frequency of male }\end{array}$ & $133.73 \pm 14.06$ & $140.39 \pm 23.06$ & $159.43 \pm 43.59$ & $196.01 \pm 65.27$ & $F=4.1, \mathrm{~S}, p=0.015$ & Normal-nodule 1-4 \\
\hline
\end{tabular}

Table 3: Comparison between normal female and diseased group of female patients

Scheffe post hoc

test, significant difference between

\begin{tabular}{llllll} 
Parameter & Normal $(n=12)$ & Polyp $(n=9)$ & Palsy $(n=10)$ & Nodule $(n=9)$ & $p$ value \\
\hline Habitual frequency & $224.45 \pm 17.71$ & $230.02 \pm 65.77$ & $198.75 \pm 60.36$ & $250.67 \pm 61.34$ & $F=1.6$, NS, $p=0.22$
\end{tabular}
of female 
Table 4: Comparison of voice quality between normal and diseased groups of patients

\begin{tabular}{|c|c|c|c|c|c|}
\hline Parameterldiagnosis & Normal $(n=23)$ & Polyp $(n=21)$ & Palsy $(n=17)$ & Nodules $(n=13)$ & $\begin{array}{l}\text { Chi-square test value, } \\
D F \text {, significance, and } \\
\text { p value }\end{array}$ \\
\hline \multirow[t]{2}{*}{ Gender } & Males = $11(47.8 \%)$ & Males = $12(57.1 \%)$ & Males $=7(41.2 \%)$ & Males $=4(30.8 \%)$ & $\begin{array}{l}\text { Chi-sq. }=2.5, \mathrm{DF}=3, \\
\mathrm{NS}, p=0.5\end{array}$ \\
\hline & Females $=12(52.2 \%)$ & Females $=9(42.9 \%)$ & Females $=10(58.8 \%)$ & Females $=9(69.2 \%)$ & \\
\hline \multirow[t]{4}{*}{ Hoarseness } & $0=15(65.2 \%)$ & $0=0(0.0 \%)$ & $0=0(0.0 \%)$ & $0=0(0.0 \%)$ & $\begin{array}{l}\text { Chi-sq. }=50.6, \mathrm{DF}=9, \\
\mathrm{~S}, p<0.001\end{array}$ \\
\hline & $1=8(34.8 \%)$ & $1=9(42.9 \%)$ & $1=4(23.5 \%)$ & $1=5(38.5 \%)$ & \\
\hline & $2=0(0.0 \%)$ & $2=4(19.9 \%)$ & $2=5(28.4 \%)$ & $2=2(15.4 \%)$ & \\
\hline & $3=0(0.0 \%)$ & $3=8(38.1 \%)$ & $3=8(47.1 \%)$ & $3=6(46.2 \%)$ & \\
\hline \multirow[t]{4}{*}{ Harshness } & $0=22(95.7 \%)$ & $0=8(38.1 \%)$ & $0=3(17.6 \%)$ & $0=4(30.8 \%)$ & $\begin{array}{l}\text { Chi-sq. }=32.3, \text { DF }=9, \\
S, p=0.001\end{array}$ \\
\hline & $1=1(4.3 \%)$ & $1=3(14.3 \%)$ & $1=5(29.4 \%)$ & $1=1(7.7 \%)$ & \\
\hline & $2=0(0.0 \%)$ & $2=1(4.8 \%)$ & $2=1(5.9 \%)$ & $2=1(7.7 \%)$ & \\
\hline & $3=0(0.0 \%)$ & $3=9(42.9 \%)$ & $3=8(47.1 \%)$ & $3=7(53.8 \%)$ & \\
\hline \multirow[t]{4}{*}{ Breathiness } & $0=12(52.2 \%)$ & $0=1(4.8 \%)$ & $0=0(0.0 \%)$ & $0=0(0.0 \%)$ & $\begin{array}{l}\text { Chi-sq. }=48.0, \mathrm{DF}=9, \\
\mathrm{~S}, p<0.001\end{array}$ \\
\hline & $1=7(30.4 \%)$ & $1=0(0.0 \%)$ & $1=1(5.9 \%)$ & $1=1(7.7 \%)$ & \\
\hline & $2=1(4.3 \%)$ & $2=1(4.8 \%)$ & $2=0(0.0 \%)$ & $2=1(7.7 \%)$ & \\
\hline & $3=3(13.0 \%)$ & $3=19(90.5 \%)$ & $3=16(94.1 \%)$ & $3=11(84.6 \%)$ & \\
\hline
\end{tabular}

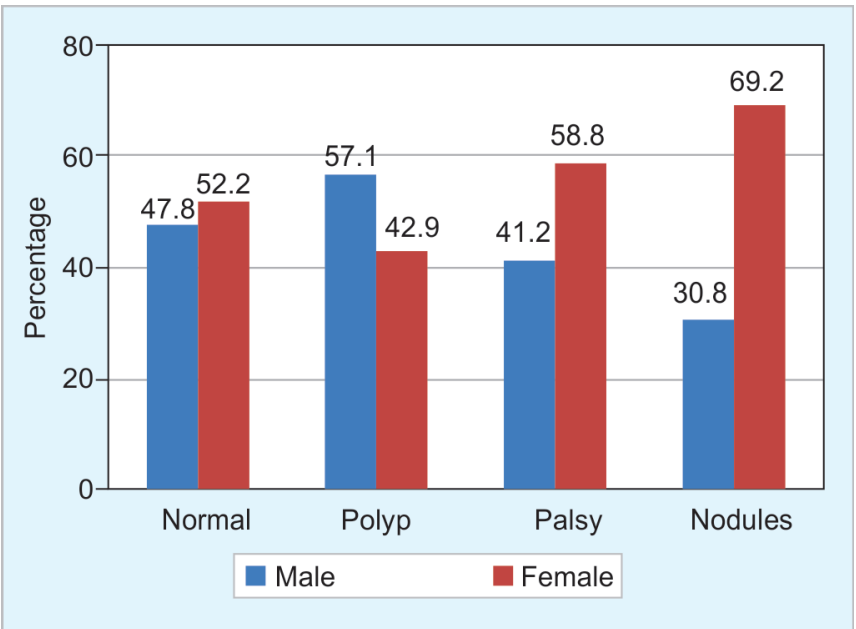

Fig. 1: Gender wise distribution (\%)

the significant difference in habitual frequency was seen between normal male and male patients with vocal cord nodule (Table 2) but, there was no significant difference between genders (Table 4 and Fig. 1).

According to Lopes et al., ${ }^{6}$ isolated acoustic measures showed poor performance $(<70 \%)$ while differentiating between patients with and without the laryngeal disorder. When analyzed in combination, the SD measures of the F0, jitter, shimmer, and GNE improved the results, reaching an accuracy of $71.76 \%$ in distinguishing between patients with and without the laryngeal disorder. Our data analysis was also done combining parameters giving significant differences.

In the study conducted by Yu et al., ${ }^{7}$ a total of 11 acoustic and aerodynamic parameters were measured. This analysis showed nine of these parameters to be relatively relevant. The appropriateness

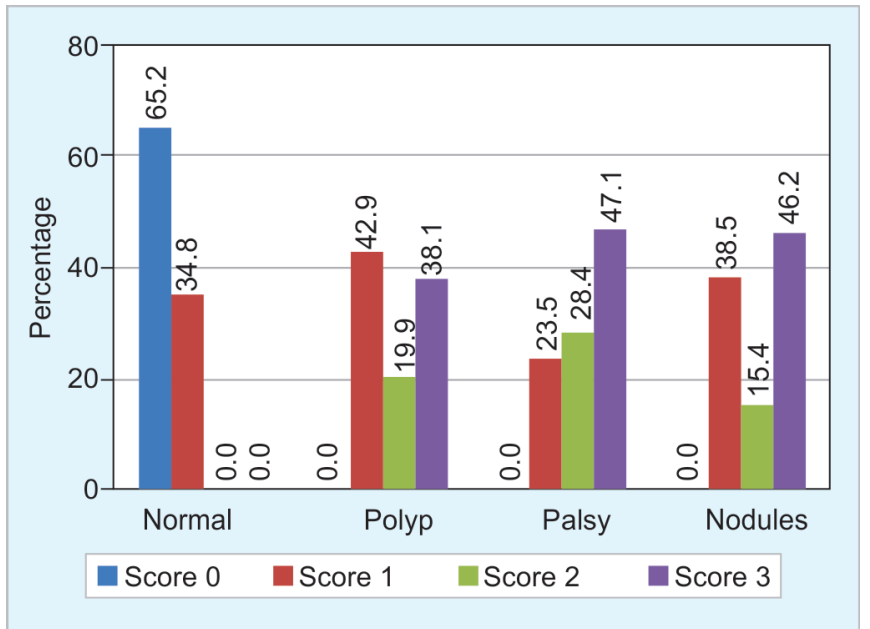

Fig. 2: Hoarseness (\%)

of these parameters was confirmed by a correlation matrix showing a significant correlation ( $p<0.01$ ). In our study, 7 of 11 parameters in vocal cord polyp and palsy whereas 8 of 11 parameters in vocal cord nodules showed significant difference.

In our data, acoustic parameters like jitter, shimmer, NNE, HNR, SNR along with voice quality scores like harsh voice, hoarse voice, and breathy voice have given statistically significant difference (Tables 1 and 4; Figs 2 to 4).

Hence, from our study, it can be said that acoustic analysis is a reliable objective tool for evaluating voice disorders.

\section{Conclusion}

Voice assessment is composed of subjective and objective methods, which help to improve clinical diagnosis and treatment of voice disorders. Among the various objective methods, parameters 


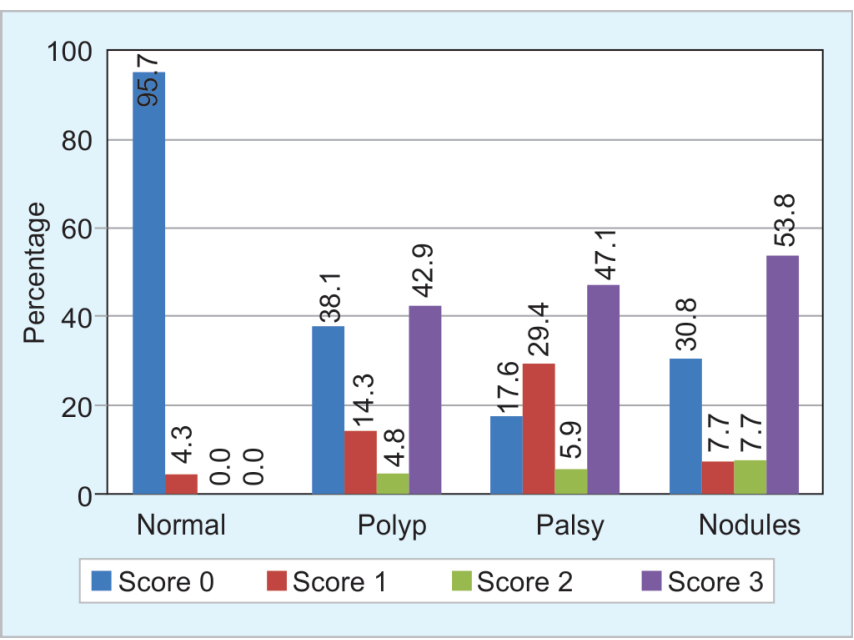

Fig. 3: Harshness (\%)

in acoustic voice analysis have given significant difference, ascertaining it as an accurate and reliable tool.

\section{References}

1. Ziwei $Y$, Zheng $P$, Pin $D$, et al. Multiparameter voice assessment for voice disorder patients: a correlation analysis between objective and subjective parameters. J Voice 2014;28(6):770-774. DOI: 10.1016/j. jvoice.2014.03.014.

2. Teixeira JP, Oliveira C, Lopes C. Vocal acoustic analysis - jitter, shimmer and HNR parameters. Proced Technol 2013;9:1112-1122. DOI: 10.1016/j. protcy.2013.12.124.

3. Teixeira JP, Gonçalves A. Algorithm for jitter and shimmer measurement sin pathologic voices. Proced Comp Sci 2016;100:271279. DOI: $10.1016 /$ j.procs.2016.09.155.

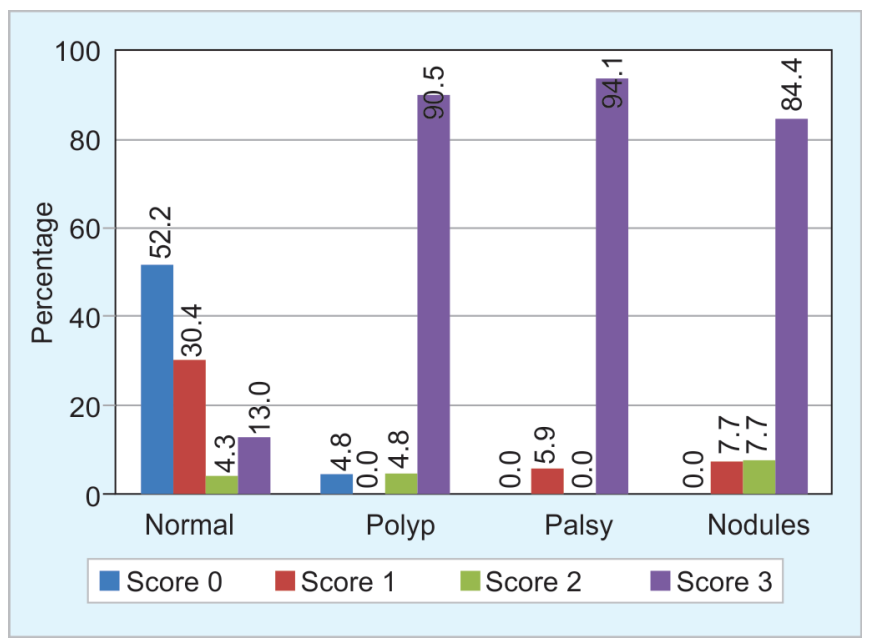

Fig. 4: Breathiness (\%)

4. Teixeira JP, Fernandes PO. Acoustic analysis of vocal dysphonia. Proced Comp Sci 2015;64:466-473. DOI: 10.1016/j.procs.2015. 08.544.

5. Teixeira JP, Fernandes PO. Jitter, shimmer and HNR classification within gender, tones and vowels in healthy voices. Proced Technol 2014;16:1228-1237. DOI: 10.1016/j.protcy.2014. 10.138.

6. Lopes LW, Batista Simões L, Delfino da Silva J, et al. Accuracy of acoustic analysis measurements in the evaluation of patients with different laryngeal diagnoses lopes. J Voice 2017;31(3):382.e15-382. e26. DOI: 10.1016/j.jvoice.2016.08.015.

7. Yu P, Ouaknine $M$, Revis J, et al. Objective voice analysis for dysphonic patients: a multiparametric protocol including acoustic and aerodynamic measurements. J Voice 2001;15(4):529-542. DOI: 10.1016/S0892-1997(01)00053-4. 